\title{
COVID-19 clinico-radiological mismatch: a proposal for a novel combined morphologic/volumetric CT severity score with blinded validation
}

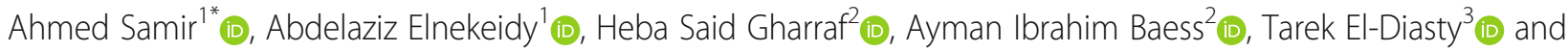 \\ Dina Altarawy ${ }^{1}$ (D)
}

\begin{abstract}
Background: Some COVID-19 patients with similar quantitative CT measurements had variable clinical presentation and outcome. The absence of reasonable clinical explanations, such as pre-existing comorbidities or vascular complications, adds to the confusion. The authors believed that neglecting the impact of certain severe morphologic features could be an alternative radiological explanation. This study aims to optimize the initial CT staging of COVID-19 and propose a new combined morphologic/volumetric CT severity index (CTSI) to solve this clinico-radiological mismatch.

Results: This multi-center study included two major steps. The first step of the study entailed a standardized combined morphologic/volumetric CT severity analyses to propose a new optimized CTSI. This was conducted retrospectively during the period from June till September 2020. It included 379 acutely symptomatic COVID-19 patients. They were clinically classified according to their oxygen saturation and respiratory therapeutic requirements into three groups: group A (mild 298/79\%), group B (borderline severity 57/15\%), and group C (severe/critical 24/6\%). The morphologic and volumetric assessment of their HRCT was analyzed according to severity, by two consultant radiologists in consensus. A new 25 point-CTSI has been created, combining eight morphological CT patterns [M1:M8; 8 points] and four grades of volumetric scores [S1:S4; 17 points]. The addition of the M5 pattern (air bubble sign), M6 pattern (early fibrosis and architectural distortion), or M7 pattern (crazy-paving) proved to increase the clinical severity. The second step of the study entailed a standardized blinded/independent validation analysis for the proposed CTSI. This was prospectively conducted on other 132 patients during October 2020 and independently performed by other two consultant radiologists. Validation results reached $80.2 \%$ sensitivity, $91.8 \%$ specificity, AUROC-curve $=$ 0.8356 , and $90.9 \%$ accuracy.

\footnotetext{
* Correspondence: Sweetjomana36@hotmail.com

'Department of Radio-diagnosis, Faculty of Medicine, Alexandria University,

Alexandria, Egypt

Full list of author information is available at the end of the article
}

\section{Springer Open}

(c) The Author(s). 2021 Open Access This article is licensed under a Creative Commons Attribution 4.0 International License, which permits use, sharing, adaptation, distribution and reproduction in any medium or format, as long as you give appropriate credit to the original author(s) and the source, provide a link to the Creative Commons licence, and indicate if changes were made. The images or other third party material in this article are included in the article's Creative Commons licence, unless indicated otherwise in a credit line to the material. If material is not included in the article's Creative Commons licence and your intended use is not permitted by statutory regulation or exceeds the permitted use, you will need to obtain permission directly from the copyright holder. To view a copy of this licence, visit http://creativecommons.org/licenses/by/4.0/. 
(Continued from previous page)

Conclusion: A new optimized CTSI with accepted validation is proposed for initial staging of COVID-19 patients, using combined morphologic/volumetric assessment instead of the quantitative assessment alone. It could solve the clinico-radiological mismatch among patients with similar quantitative CT results and variable clinical presentation during the absence of pre-existing comorbidities or vascular complications.

Keywords: COVID-19, CTSI, Morphologic, Volumetric, Quantitative

\section{Background}

On March 12, WHO announced COVID-19 as a pandemic $[1,2]^{\cdot}$ Progression to ARDS and coagulation dysfunction was reported in severe cases [3] Chest computed tomography $(\mathrm{CT})$ plays an important role in the initial evaluation and follow up of COVID-19 patients because of its high sensitivity [4-6]. The CT findings may also precede the onset of symptoms while the PCR screening may show false-negative results [7]. The European Society of Radiology and the European Society of Thoracic imaging recommended CT pulmonary angiography (CTPA) when non-enhanced CT failed to explain the severity of the respiratory failure [8].

Several CT severity scores were introduced for COVID-19; all were based on manual or automated quantitative evaluation alone. Meanwhile, some COVID19 patients with similar quantitative CT measurements had variable clinical presentation and outcome. The absence of any clinical explanations, such as pre-existing comorbidities or vascular complications, adds to this confusion.

The authors believed that neglecting the impact of certain severe morphologic features could be an alternative radiological explanation for this mismatch and suggested that the combination between volumetric and morphologic assessment is mandatory. Consequently, the authors performed this study to optimize COVID-19 initial CT staging and propose a new combined morphologic/volumetric CT severity index (CTSI) to solve the clinico-radiological mismatch among patients without comorbidities or vascular complications.

\section{Methods}

\section{Study protocol and human population}

This multi-center study was collectively conducted on 511 acutely symptomatic patients proved with COVID19 during the period from June till October 2020. It was approved by the Institutional Ethics Committee. Patient consent was waived by the Research Ethics Board, assuring the respect of the confidentiality of patient's data and medical records. The manuscript has no overlap with any previously published work.

Inclusion criteria were acutely symptomatic COVID-19 patients (during the 10 days from the onset of first clinical complaint) with positive PCR results and complete medical records.

Exclusion criteria were (1) asymptomatic patients, (2) incomplete medical records, (3) degraded quality of CT images with respiratory motion artifacts, (4) patients with known explanation for the clinicalradiological mismatch (48 patients were already excluded before the onset of the study) such as (A) pre-existing cardio-pulmonary or extra-pulmonary comorbidities such as emphysema, interstitial lung diseases, lung cancer, morbid obesity, co-existing neurological, cardiac, or abdominal diseases. All can impact the patient original $\mathrm{O}_{2}$ saturation and yield more deterioration of his condition not proportionate to the degree of lung involvement. (B) Vascular complications proved by CT pulmonary angiography among borderline-severe or severe/critical patients, such as acute pulmonary embolism.

\section{Clinical evaluation}

The clinical evaluation was performed by two consultant pulmonologists (having long time experience in the field of chest diseases; $18-20$ years).

Patients were clinically classified according to the clinical symptoms, oxygen saturation, and respiratory therapeutic requirements into three groups [9]:

- Group [A] (mild): patients with 95-100\% $\mathrm{O}_{2}$ saturation/room-air (RA), absent or type I dyspnea, respiratory rate $(R R)<30 / \mathrm{min}$, and no need for $\mathrm{O}_{2}$ support.

- Group [B] (borderline severe): patients with 93-94\% $\mathrm{O}_{2}$ saturation/RA plus type II or III dyspnea and/or tachypnea ( $R R \geq 30 / \mathrm{min}$ ) or both.

- Group [C] (severe/critical): patients with $<93 \% \mathrm{O}_{2}$ saturation/RA up to ARDS.

The patients in group [B] and [C] could be indicated for initial high flow nasal oxygen therapy up to mechanical ventilation.

\section{CT machines and scanning parameters:}

Multiple MDCT machines were used: (1) SOMATOM Sensation 64, Siemens Medical Systems, Germany, (2) Canon Medical Systems; Toshiba Aquilion 64, USA, and 
(3) Canon Medical Systems; Toshiba Aquilion CXL/CX 128, USA.

CT scanning parameters were slice thickness: 1$1.25 \mathrm{~mm}$, tube rotation: $0.6-0.9 \mathrm{~s}$, detector collimation $1 \mathrm{~mm}, 120-130 \mathrm{kVp}$, and $200 \mathrm{~mA}$, FOV $=350$ $\mathrm{mm} \times 350 \mathrm{~mm}$. Intravenous contrast administration was not used.

\section{Study design and steps}

The study included two major steps:

(1) First step: standardized combined morphological/ volumetric CT severity analyses and proposal of a new CTSI.

(2) Second step: standardized blinded/independent validation analysis for the proposed new CTSI.

\section{Step (1): standardized combined morphological/volumetric CT severity analyses and proposal of a new CTSI}

It was conducted retrospectively during the period from June till September 2020. It included 379 acutely symptomatic COVID-19 patients. They were 242 males and 137 females (63.9\%:36.1\%). Their age ranged from 10 to 80 years (mean age $45.42 \pm 20.1 \mathrm{SD}$ ). They were clinically classified into three groups: Group [A] (mild 298/79\% patients). Group [B] (borderline severity 57/ $15 \%$ patients). Group [C] (severe or critical 24/6\% patients).

CT images were analyzed in consensus with the availability of medical records by two expert consultant radiologists (having long time experience in thoracic imaging; 15 and 25 years). They performed combined CT volumetric and morphologic assessment followed by severity analysis and a new combined CTSI proposal.

[I] Volumetric/quantitative assessment [size of lesions (S)]

OsiriX MD 11.0 software (Pixmeo SARL, Geneva, Switzerland) was utilized for imaging review and volumetric/quantitative assessment of all patients, so the variability of the used MSCT machines would not impact the quantitative assessment. It was utilized for automated calculation of the total and pathological lung volumes based on threshold interval adjustment during the region of interest (ROI) 2D/3D color-coded reconstruction. (0:- $1024 \mathrm{Hu})$ the interval was set for total lung volume calculation and $(0:-700 \mathrm{Hu})$ interval was almost set for pathological lung volume calculation. Lesions were classified into four grades:

- [S1] Patchy lesions involving ( $<15 \%$ of lung volume),
- [S2] Patchy lesions involving (15-25\% of lung volume),

- [S3] Patchy lesions involving (25-50\% of lung volume),

- [S4] Diffuse lung involvement (> 50\% of lung volume).

[II] Morphologic assessment (M)

Eight morphologic HRCT patterns were traced:

- [M1] Pure ground-glass opacities (GGOs) or solid nodules with peri-focal GG (halo sign).

- [M2] GGOs with a peripheral organization "Atoll or reversed halo sign."

- [M3] GGOs mixed with consolidative changes.

- [M4] Homogeneous or "curvilinear" consolidations; the latter is parallel to the pleural lining.

- [M5] GGOs with "air bubble sign"; are small airfilled spaces within the ground-glass or consolidative changes, representing a cut section of sub-segmental bronchiolectasis sequel to fibrosis with focal air trapping.

- [M6] GGOs with "early secondary fibrotic changes" and architectural distortion; are irregular linear streaks and atelectatic plates, representing early scarring of cellular components, which could be associated with bronchial wall thickening and bronchiectatic changes.

- [M7] Patchy GGOs with smooth septal thickening "crazy-paving pattern,"

- [M8] Diffuse alveolar damage (DAD) pattern, showing diffuse lung involvement with either of the above-mentioned CT patterns or mixed (notably crazy-paving pattern and air bubble sign).

Extra-parenchymal HRCT findings were also assessed, including significant nodal enlargement (short axis $>1$ $\mathrm{cm}$ ) and pleuro-pericardial effusions.

The overall time for this combined quantitative and morphologic $\mathrm{CT}$ assessment was estimated and ranged from 6 to $12 \mathrm{~min}$.

[III] Statistical severity analyses and CTSI proposal

- Prevalence of HRCT characteristics among the total number of patients.

* Statistical analysis of significant relation between each HRCT volumetric or morphologic characteristic and clinical severity. Chi-square tests and $P$ value measurements were performed using an online calculator (https://www. 
socscistatistics.com). $P$ value $(<0.05)$ was considered statistically significant.

* Calculation of the severity ratio for each HRCT abnormality among each group from the following equation [Severity ratio $=$ Prevalence rate of HRCT finding among the total number of each patients' group/Prevalence rate of each patients' group among total sample size]. The total severity index is then summed and the CTSI model is created.

\section{Step (2): standardized blinded/independent validation analysis for the proposed new CTSI}

It was conducted prospectively during October 2020 on other 132 patients. They included 72 males and 60 females (54.5\%:45.5\%). Their age ranged from 22 to 67 years (mean age $41.13 \pm 11.69 \mathrm{SD}$ ). CT images were analyzed independently by two expert consultant radiologists (having long time experience in thoracic imaging;
10 and 30 years). They were blinded from the clinical records.

The following statistical methods were utilized:

* Inter-observer agreement (IOA) using Cohen's Kappa test.

* Prevalence, sensitivity, specificity, positive predictive value (PPV), and negative predictive value (NPV), using an online diagnostic test calculator (http:// araw.mede.uic.edu/cgi-bin/testcalc.pl).

* Receiver operating characteristics (ROC) curve, area under the curve (AUC), confidence interval, and accuracy, using QI Macros 2020 Excel software.

\section{Results}

A flow diagram is demonstrating the study methodology including patients' selection, study design, and steps together with final clinical and radiological results (Fig. 1).

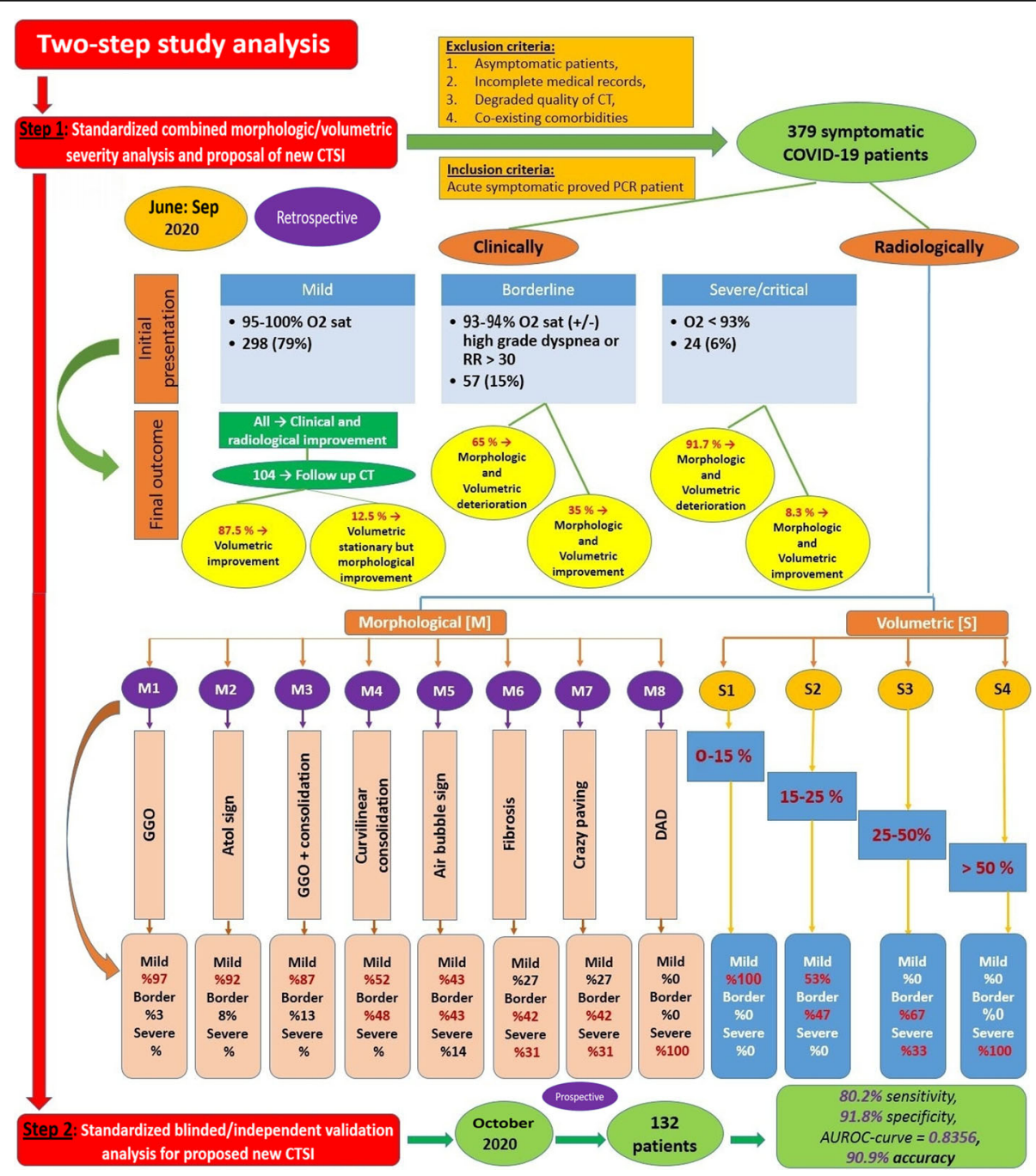

Fig. 1 A flow diagram design summarizing the study steps and results 


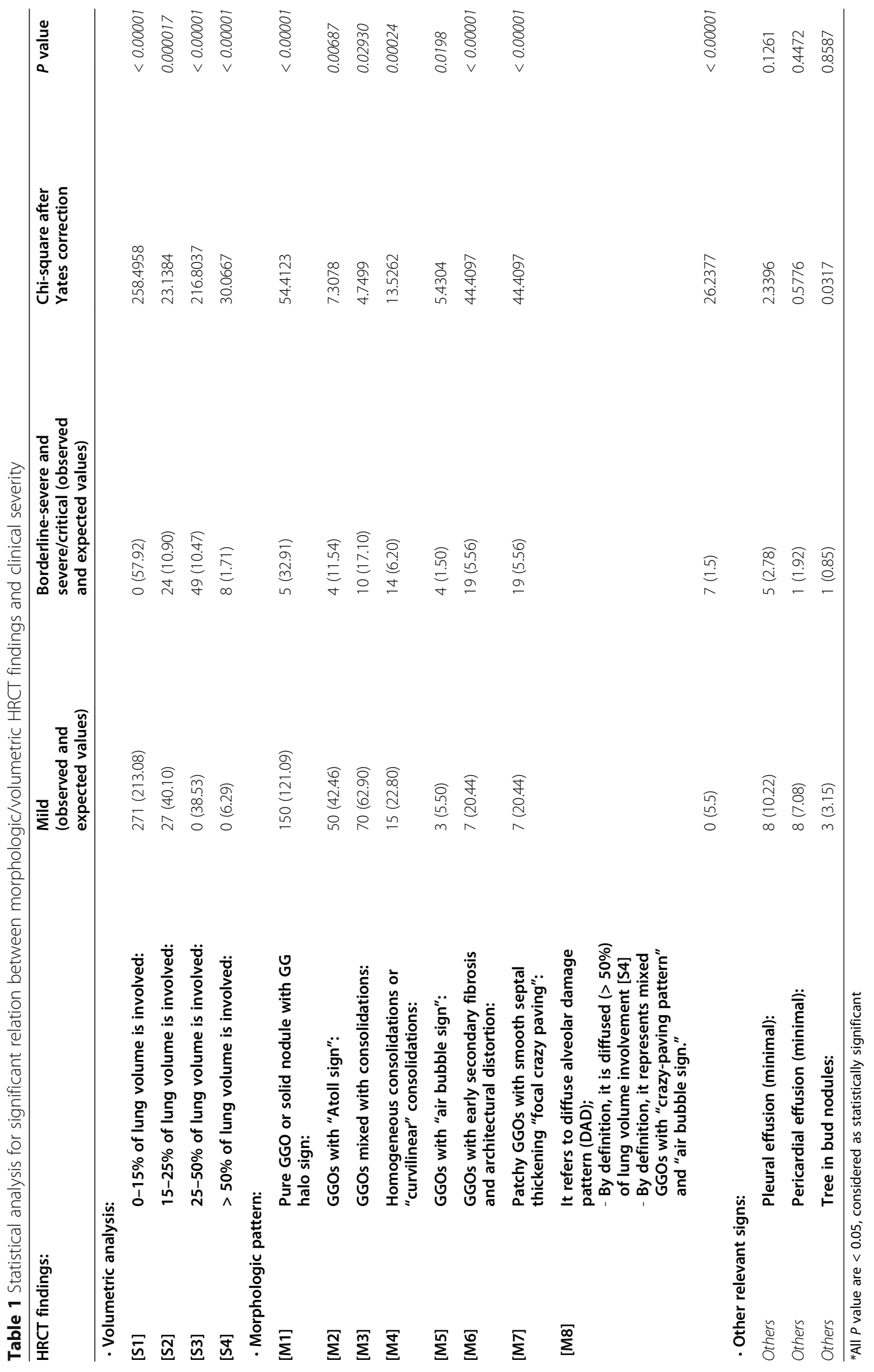




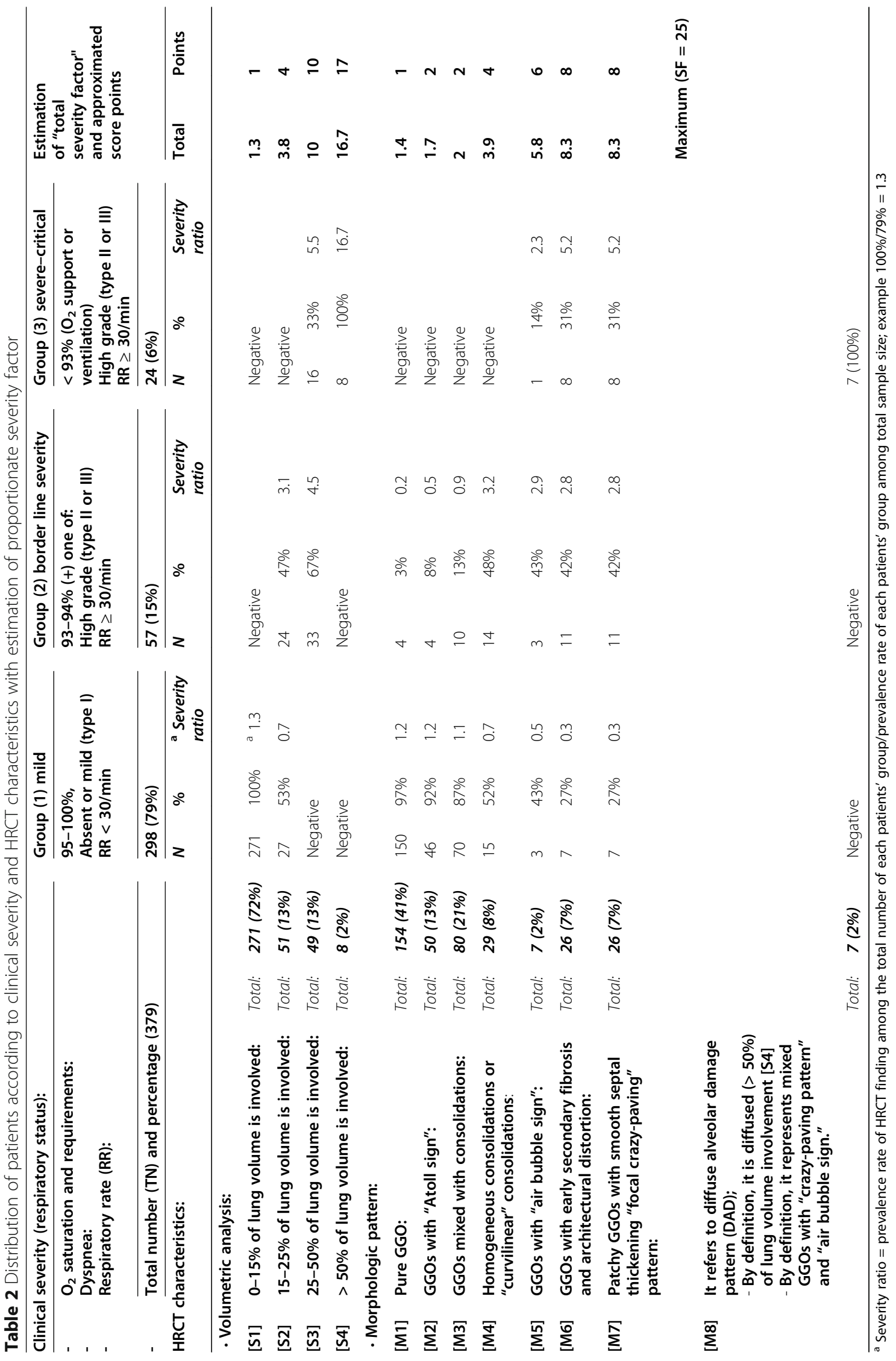


Step (1): standardized combined morphological/ volumetric CT severity analyses and proposal of a new CTSI

\section{Size of the lesions [S] with volumetric/quantitative} assessment

A significant relation between the size of the lesions and clinical severity was statistically proved $(P$ values ranged from 0.00017 to $<0.0001$ ) (Table 1). Prevalence and severity analysis was performed and detailed in (Table 2). [S1] lesions were the highest prevalent $(72 \%)$ and the least severe (1.3). On the other hand, [S4] lesions were the least prevalent $(2 \%)$ and the most severe (16.7). Examples of volumetric assessment are demonstrated (Fig. 2).

\section{Morphological CT patterns [M]}

A significant relation between morphological HRCT patterns and clinical severity was statistically proved $(P$ values ranged from 0.02930 to $<0.0001$ ) (Table 1 ). Prevalence and severity analysis was performed and detailed in (Table 2). [M1] lesions were the most prevalent (41\%) and the least severe (1.4). On the other hand, [M5] lesions were the least prevalent (2\%) and [M6] with [M7] lesions were the most severe (8.3 each).

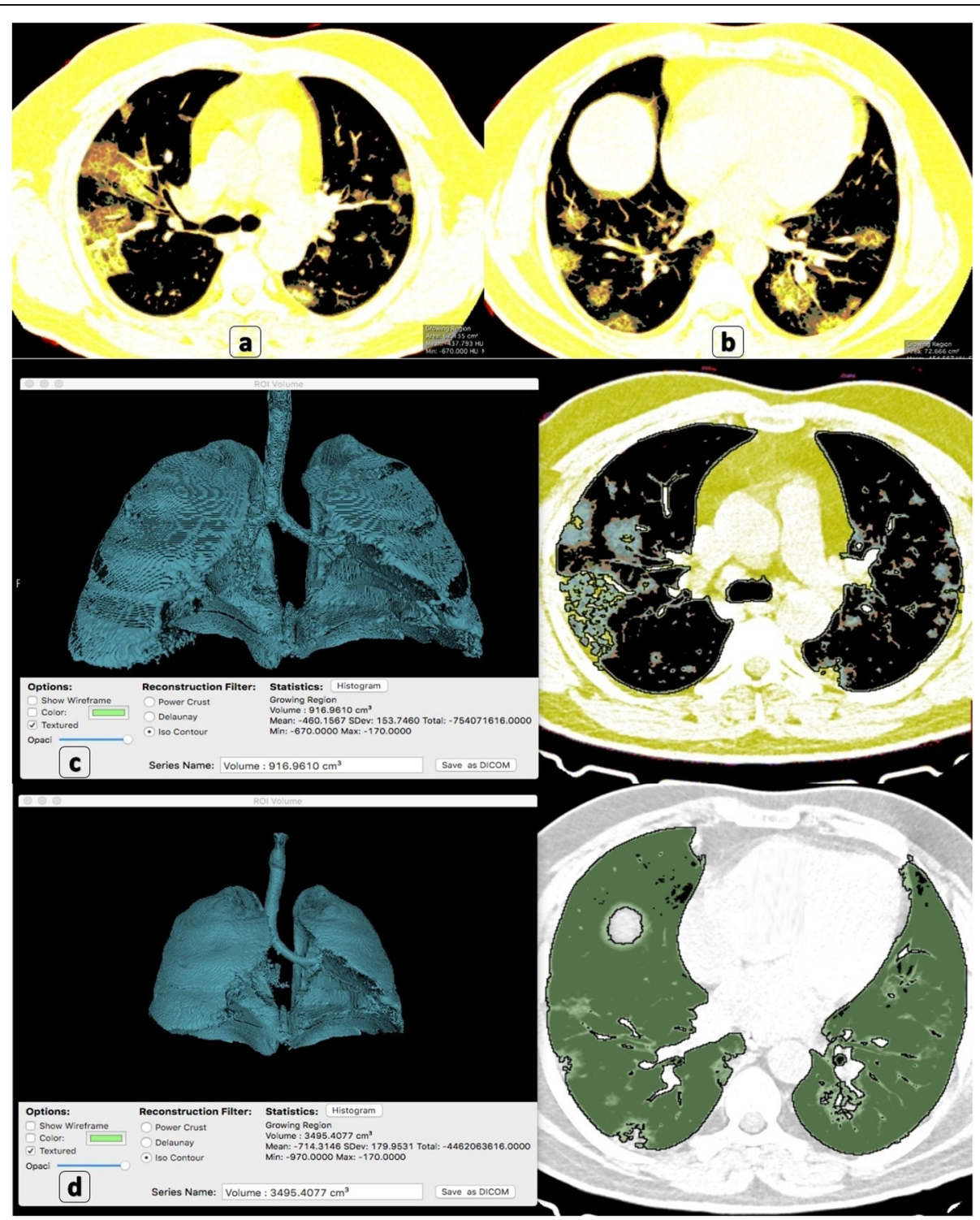

Fig. 2 Volumetric/quantitative assessment using OsiriX MD 11.0 software: A 57-year-old male patient proved with COVID-19. a, b Osirix 2D computed volumetric analysis (axial cuts) show bilateral lower lobar sub-pleural patchy GGOs with "crazy-paving" reaching peri-hilar zones proximally. c Osirix 3D computed volumetric analysis after threshold interval adjustment shows $917 \mathrm{~cm}^{3}$ affected volume of the lung. d Osirix 3D computed volumetric analysis after threshold interval adjustment shows $3495 \mathrm{~cm}^{3}$ total lung volume 
Trivial percentages of other extra-parenchymal CT signs were depicted and detailed in (Table 1). No significant relation to clinical severity could be statistically proved ( $P$ values ranged from 0.1261 to 0.8587 ).

\section{Combined volumetric and morphologic analysis}

All patients with $>50 \%$ lung involvement [S4] were clinically severe or critical. Around $67 \%$ of patients with 25-50\% lung involvement [S3] were borderline, while the remaining $33 \%$ were clinically severe or critical because of the additive effect of either [M6] pattern (early patchy fibrosis with architectural distortion) or [M7] pattern (patchy GGOs with crazy-paving pattern) (Figs. 3 and 4).
All patients with $<15 \%$ lung involvement [S1] were mild. Around $53 \%$ of patients with $15-25 \%$ lung involvement [S2] were mild, while the remaining $47 \%$ were borderline because of the additive effect of the [M5] pattern (GGOs with air bubble sign) (Fig. 5)

The overall time for this combined quantitative and morphologic CT assessment was estimated and ranged from 6 to $12 \mathrm{~min}$.

\section{A proposed new descriptive $C T$ coding}

Based on the combination of morphologic and volumetric analysis, a new descriptive CT code was proposed $[M$

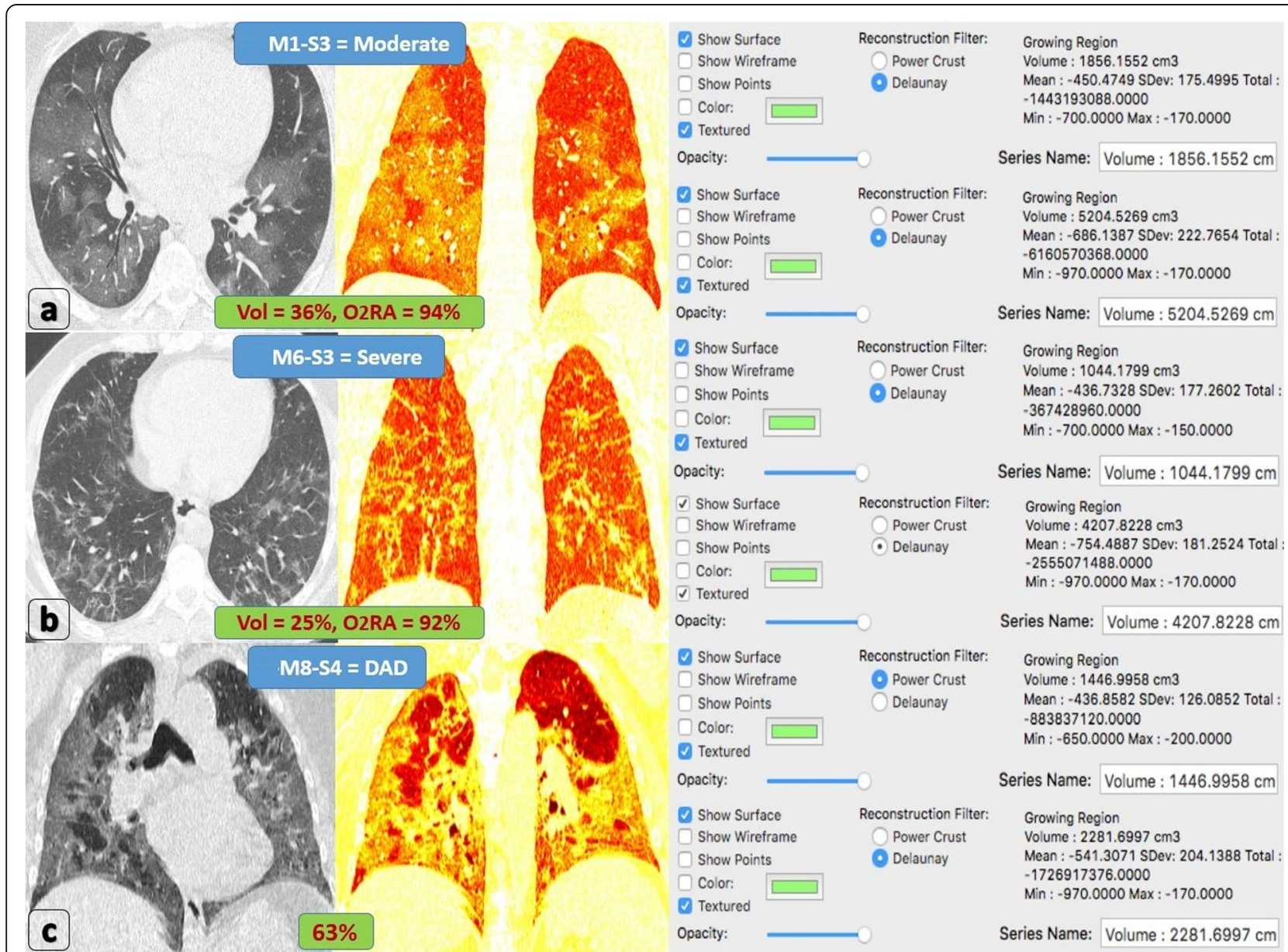

Fig. 3 Combined morphologic/volumetric analysis. a A 33-year-old male patient proved with COVID-19 complaining of dyspnea, tachypnea, and $94 \% \mathrm{O}_{2} \mathrm{RA}$ (consistent with border-line severity). Axial CT lung window and coronal 2D computed volumetric analysis showing bilateral widespread patchy pure GGOs. 3D computed volumetric analysis after threshold interval adjustment revealed 1856 cc pathological lung volume, 5204 cc total lung volume, and 36\% lung involvement. Overall CT coding (M1-S3). b A 61-year-old male patient proved with COVID-19 complaining of dyspnea, tachypnea, and $92 \% \mathrm{O}_{2}-\mathrm{RA}$ (clinically severe). Axial CT lung window and coronal 2D computed volumetric analysis show bilateral patchy GGOs mixed with early fibrotic changes and mild parenchymal distortion. 3D computed volumetric analysis after threshold interval adjustment revealed 1044 cc pathological lung volume, 4207 cc total lung volume and 25\% lung involvement. Overall CT coding (M6-S3). c A 67-year-old male patient proved with COVID-19 complaining of severe dyspnea and 82\% $\mathrm{O}_{2} \mathrm{RA}$ (critical patient indicated for intubation). Coronal CT lung window and coronal 2D computed volumetric analysis showing bilateral diffuse lung involvement with GGOs and super-added septal thickening "crazy-paving pattern." 3D computed volumetric analysis after threshold interval adjustment revealed $1446 \mathrm{cc}$ pathological lung volume, 2281 cc total lung volume, and 64\% lung involvement. Overall CT coding: M8-S4 = DAD 


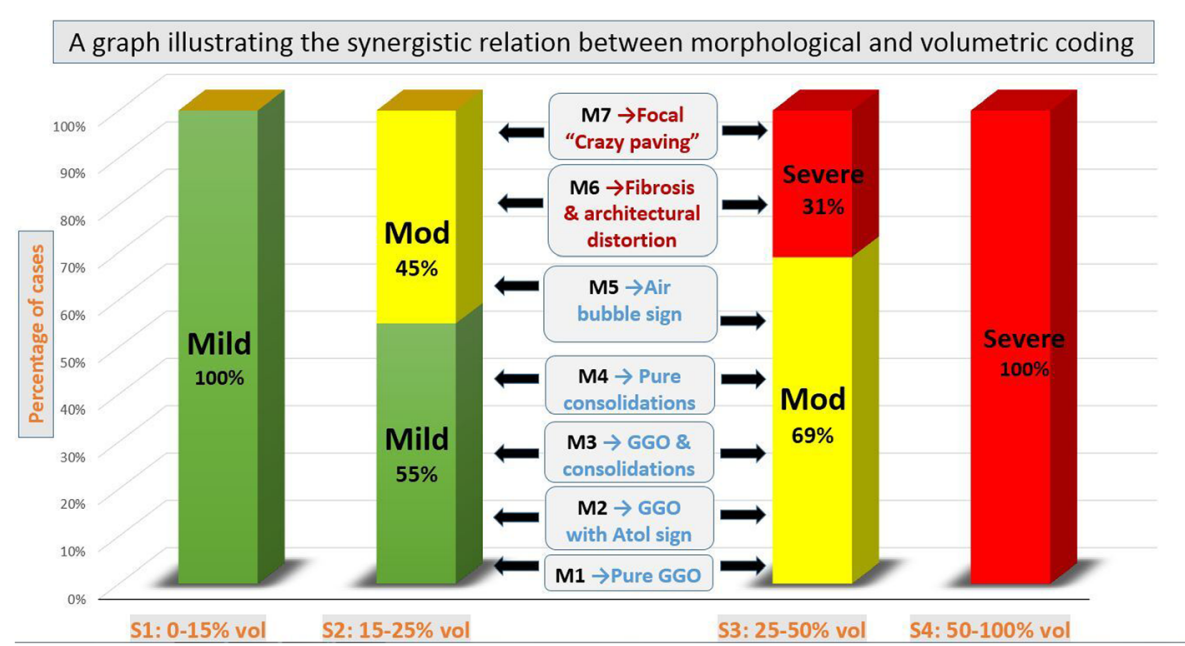

Fig. 4 A graph illustrating the additive relation between morphologic and volumetric coding: 100\% of [S1] patients are mild whatever their morphological pattern. 100\% of [S4] patients are severe whatever their morphological pattern. While 55\% of [S2] patients are mild, the remaining $45 \%$ are moderate because of the additive effect of [M5, M6 or M7] patterns. While $67 \%$ of [S3] patients are moderate, the remaining $33 \%$ are severe because of the additive effect of [M6 and M7] patterns

$(1-8) / S(1-4)]$. Distribution prevalence for our patients regarding new CT coding was grouped (Table 3).

\section{A proposed new optimized CTSI (Table 4)}

Based on the prevalence and severity analysis, an optimized CTSI is proposed with a " $2-25$ points" scoring system (Table 4); (1-8 points) are given for the morphologic assessment $[\mathrm{M}]$, while (1-17 points) are given for the volumetric/size score [S]. Three categories were achieved corresponding to the clinical severity: (1) mild CTSI (2-9 points), (2) borderline or moderate CTSI (10-17 points), and (3) severe or critical CTSI (18-25 points). A survey for patients regarding new CT coding and new CTSI was demonstrated (Figs. 5 and 6).

\section{Evaluation of patient clinical-radiological outcome (14 to 30} days after the onset of symptoms)

All patients with mild clinical presentation showed clinical improvement over the next 14-21 days. Only 104/ 298 patients (35\%) with delayed recovery had followed up CT scans. Radiological improvement was noted among all of them regarding the morphological CT features (depicted by resolution or regression in the density of ground glass or consolidation lesions). Around 91/104 patients $(87.5 \%)$ also showed regression in quantitative measurements, while $13 / 104$ patients (12.5\%) showed the stationary course of which, but with morphological improvement.

$37 / 57$ patients $(65 \%)$ with borderline severity had a follow-up assessment with CT pulmonary angiography protocol after the deterioration of clinical condition into severe/critical status $\left(\mathrm{O}_{2}\right.$ saturation dropped below $\left.93 \%\right)$. The radiological progressive course was depicted regarding both quantitative measurements and morphological features of parenchymal fibrosis and crazy paving pattern. One patient who had a previous "air bubble sign" developed pneumothorax. The vascular assessment was unremarkable. Thirty-one patients received high flow nasal oxygen while 6 patients needed mechanical ventilation later on.

$22 / 24$ critical patients $(91.7 \%)$ showed deterioration in the clinical condition regarding the $\mathrm{O}_{2}$ requirements; 20 patients needed mechanical ventilation while two patients had sudden death before ventilation. Only bedside X-ray was used for follow-up of these patients. Two patients only had partial improvement regarding $\mathrm{O}_{2}$ saturation and requirements and follow-up CT scans later on revealed regression regarding both quantitative and morphological CT features.

\section{Step (2): standardized blinded/independent validation analysis for the proposed new CTSI}

Accepted inter-observer agreement (IOA) was achieved (ranging from 81.3\%:100\%). High accuracy for the proposed new CTSI was reached (ranging from 90.1\%:91.7\%). High degrees of specificity and NPV were estimated (ranging from $86.8 \%: 96.4 \%$ and $80.5 \%: 97.2 \%$, respectively). Moderate to a high degree of sensitivity and PPV were estimated (ranging from 66.7\%:91.5\% and 60.9\%:94.5\%, respectively). Based on AUROC curve measurements (ranging from 0.7765: 0.9003 ) and confidence intervals (ranging from 0.684: 0.832), the proposed CTSI expressed excellent, good, 


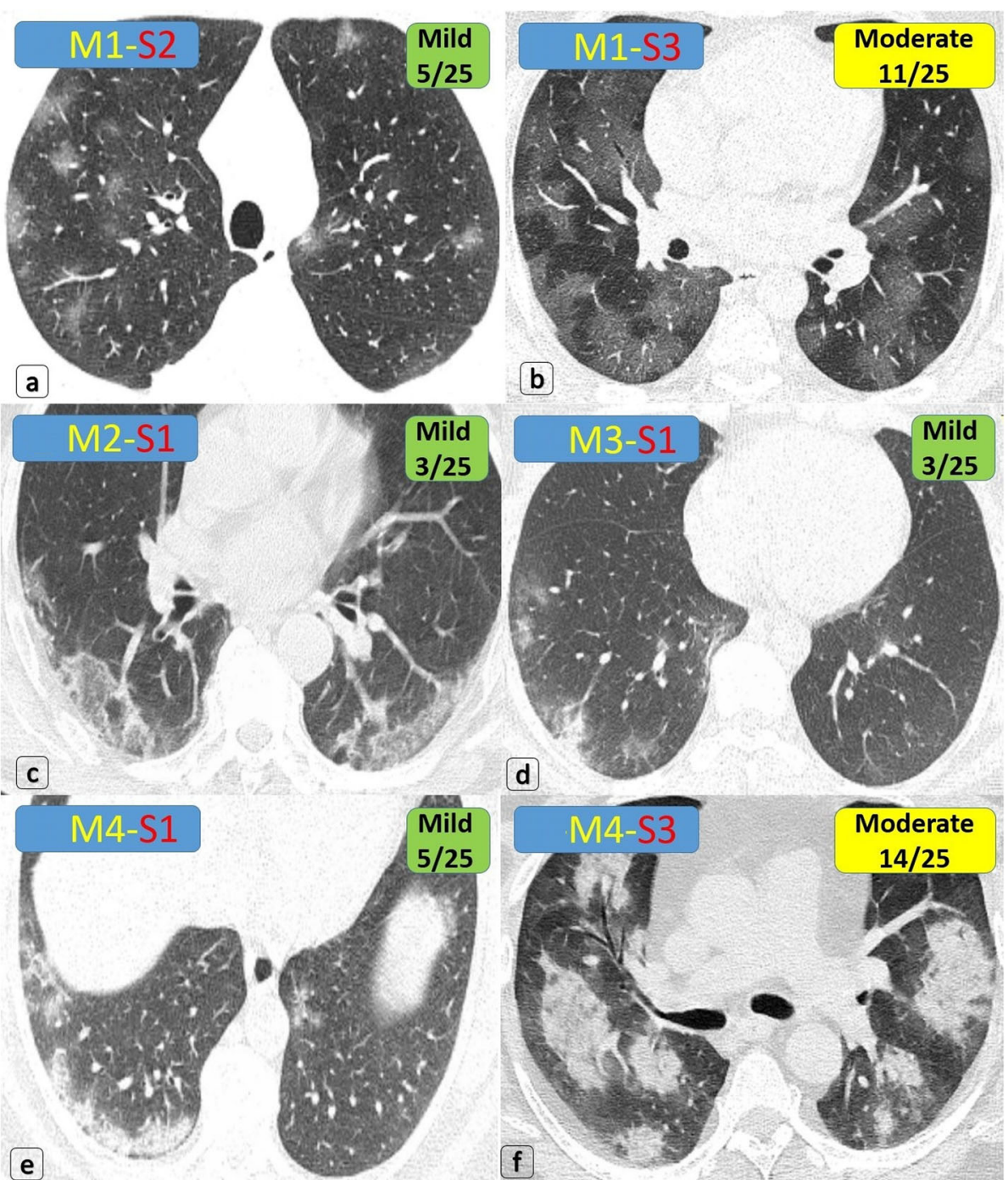

Fig. 5 Multiple patients proved with COVID-19, having variable bilateral mild or moderate/border-line lung involvement. a Multiple (> 3 in number) small $(<3 \mathrm{~cm}$ ) bilateral sub-pleural and to lesser extent proximal pure GG nodules (around 5\% of lung involvement) ... COV [M1-S2] ... CTSI [5/25] ... Mild degree. b Bilateral lower lobar large pure ground-glass patches (around 36\% of lung involvement) ... COV [M1-S3] ... CTSI [11/25] ... Moderate degree. $\mathbf{c}$ Bilateral lower lobar sub-pleural pure ground-glass patches ( $>3 \mathrm{~cm}$ in size but only $2 \mathrm{~cm}$ deep from the pleural lining) with peripheral "Atoll sign" (around 4\% of lung involvement) ... COV [M2-S1] ... CTSI [3/25] ... Mild degree. d Bilateral scattered small $(<3 \mathrm{~cm}) \mathrm{GGOs}$ are seen; one of them is mixed with consolidative changes in the right lower lobe (around 3\% of lung involvement) ... COV [M3-S1] ... CTSI [3/25] ... Mild degree. e Right lower lobar curvilinear pattern of consolidations ( $>3 \mathrm{~cm}$ in size but only $1 \mathrm{~cm}$ deep from the pleural lining), a small left lower lobar GGO is seen (around 3\% of lung involvement) ... COV [M4-S1] ... CTSI [5/25] ... Mild degree. f Bilateral multiple variably sized proximal and peripheral (>3 cm) homogeneous consolidative patches (around 27\% of lung volume) ... COV [M4-S3] ... CTSI [14/25] ... Moderate degree

and fair validity for evaluation of mild, borderline, and severe patients, respectively. A detailed review of all validation analysis measurements was grouped (Table 5) and demonstrated (Fig. 7).

\section{Discussion}

Clinical confusion was aroused about COVID-19 patients who had similar quantitative CT measurements and variable clinical prognosis. The presence of preexisting comorbidities or vascular angiopathy is known clinical and radiological explanations but they could be absent. The authors suggested another radiological explanation by adding morphological severity to the volumetric severity.

This study was different from previous studies which only stressed the quantitative lung assessment and did not exclude those confounding parameters; such as Yang et al. [10], Lessmann et al. [11], and Li et al. [12]. Leonardi et al. [13] excluded only pleuro-parenchymal lung diseases. On the other hand, authors agreed with 


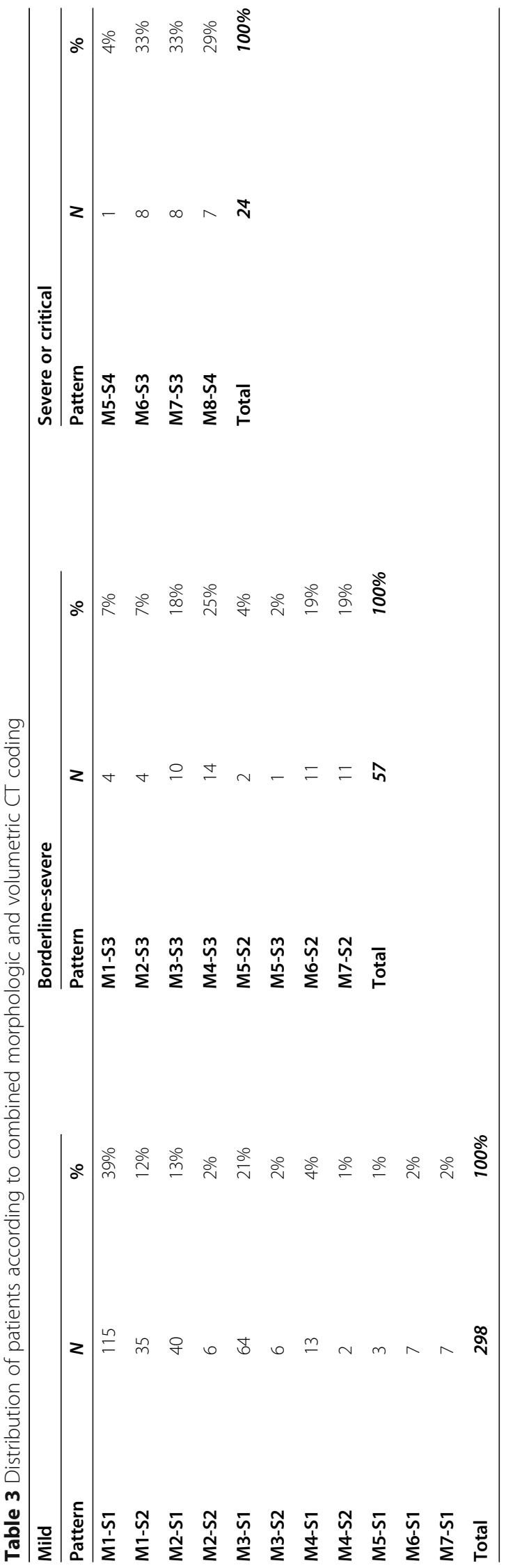


Table 4 Combined morphologic/volumetric CT coding - new proposed CTSI for COVID-19

\begin{tabular}{|c|c|c|c|c|c|}
\hline \multicolumn{4}{|c|}{ Morphologic/Volumetric CT Coding: } & Score/25 & Grade \\
\hline \multirow[t]{5}{*}{ M1 } & \multicolumn{5}{|c|}{ Pure GGO: (1 Point) } \\
\hline & s1 & $0-15 \%$ of lung volume is involved. & "1 Point" & $2 / 25$ & Mild \\
\hline & s2 & $15-25 \%$ of lung volume is involved. & "4 Points" & $5 / 25$ & Mild \\
\hline & s3 & $25-50 \%$ of lung volume is involved. & "10 Points" & $11 / 25$ & Borderline severity \\
\hline & s4 & $>\mathbf{5 0} \%$ of lung volume is involved. & "17 Points" & $18 / 25$ & Severe \\
\hline \multirow[t]{5}{*}{ M2 } & \multicolumn{5}{|c|}{ GGOs with "Atoll sign": (2 Points) } \\
\hline & s1 & $0-15 \%$ of lung volume is involved. & "1 Point" & $3 / 25$ & Mild \\
\hline & s2 & $15-25 \%$ of lung volume is involved. & "4 Points" & $6 / 25$ & Mild \\
\hline & s3 & $25-50 \%$ of lung volume is involved. & "10 Points" & $12 / 25$ & Borderline severity \\
\hline & s4 & $>50 \%$ of lung volume is involved. & "17 Points" & $19 / 25$ & Severe \\
\hline \multirow[t]{5}{*}{ M3 } & \multicolumn{5}{|c|}{ GGOs with consolidations: (2 Points) } \\
\hline & s1 & $0-15 \%$ of lung volume is involved. & "1 Point" & $3 / 25$ & Mild \\
\hline & s2 & $15-25 \%$ of lung volume is involved. & "4 Points" & $6 / 25$ & Mild \\
\hline & s3 & $25-50 \%$ of lung volume is involved. & "10 Points" & $12 / 25$ & Borderline severity \\
\hline & s4 & $>\mathbf{5 0} \%$ of lung volume is involved. & "17 Points" & $19 / 25$ & Severe \\
\hline \multirow[t]{5}{*}{ M4 } & \multicolumn{5}{|c|}{ Homogenous consolidations or "Curvilinear" consolidations: (4 Points) } \\
\hline & s1 & $0-15 \%$ of lung volume is involved. & "1 Point" & $5 / 25$ & Mild \\
\hline & s2 & $15-25 \%$ of lung volume is involved. & "4 Points" & $8 / 25$ & Mild \\
\hline & s3 & $25-50 \%$ of lung volume is involved. & "10 Points" & $14 / 25$ & Borderline severity \\
\hline & s4 & $>50 \%$ of lung volume is involved. & "17 Points" & $21 / 25$ & Severe \\
\hline \multirow[t]{5}{*}{ M5 } & \multicolumn{5}{|c|}{ GGOs with "Air bubble sign": (6 Points) } \\
\hline & s1 & $0-15 \%$ of lung volume is involved. & "1 Point" & $7 / 25$ & Mild \\
\hline & \$2 & $15-25 \%$ of lung volume is involved. & "4 Points" & $10 / 25$ & Borderline severity \\
\hline & s3 & $25-50 \%$ of lung volume is involved. & "10 Points" & $16 / 25$ & Borderline severity \\
\hline & s4 & $>\mathbf{5 0} \%$ of lung volume is involved. & "17 Points" & 23/25 & Severe \\
\hline \multirow[t]{5}{*}{ M6 } & \multicolumn{5}{|c|}{ GGOs \& secondary fibrosis with architectural distortion (8 Points) } \\
\hline & s1 & $0-15 \%$ of lung volume is involved. & "1 Point" & 9/25 & Mild \\
\hline & s2 & $15-25 \%$ of lung volume is involved. & "4 Points" & $12 / 25$ & Borderline severity \\
\hline & s3 & $25-50 \%$ of lung volume is involved. & "10 Points" & $18 / 25$ & Severe \\
\hline & $\mathbf{S 4}$ & $>50 \%$ of lung volume is involved. & "17 Points" & $25 / 25$ & Severe \\
\hline \multirow[t]{4}{*}{ M7 } & \multicolumn{5}{|c|}{ Patchy GGOs with smooth septal thickening "Focal crazy paving" pattern (8 Points) } \\
\hline & s1 & $0-15 \%$ of lung volume is involved. & "1 Point" & 9/25 & Mild \\
\hline & s2 & $15-25 \%$ of lung volume is involved. & "4 Points" & $12 / 25$ & Borderline severity \\
\hline & s3 & $25-50 \%$ of lung volume is involved. & "10 Points" & $18 / 25$ & Severe \\
\hline M8 & \multicolumn{3}{|c|}{$\begin{array}{l}\text { It refers to diffuse alveolar damage pattern (DAD); } \\
-\quad \text { By definition it is diffuse (> 50\%) of lung volume involvement [S4] } \\
-\quad \text { By definition it represent mixed GGOs with "Crazy-paving pattern" and "Air-bubble sign". }\end{array}$} & $25 / 25$ & Severe \\
\hline & & Mild: $2-9$ & Borderline severity $(10-17)$ & re or $\mathrm{C}$ & al (18-25) \\
\hline
\end{tabular}

studies that pointed to the impact of some morphological patterns on clinical severity, such as $\mathrm{Li}$ et al. [14] and Zhao et al. [15], but they did not correlate them to CT severity scoring. Qin et al. [16] put isolated quantitative severity score beside dedicated severity score for "crazy paving pattern" and consolidations.

In this study, all patients with $>50 \%$ lung involvement were clinically severe/critical. This is precisely keeping with $\mathrm{Li}$ et al. [12] and generally matching with previous 


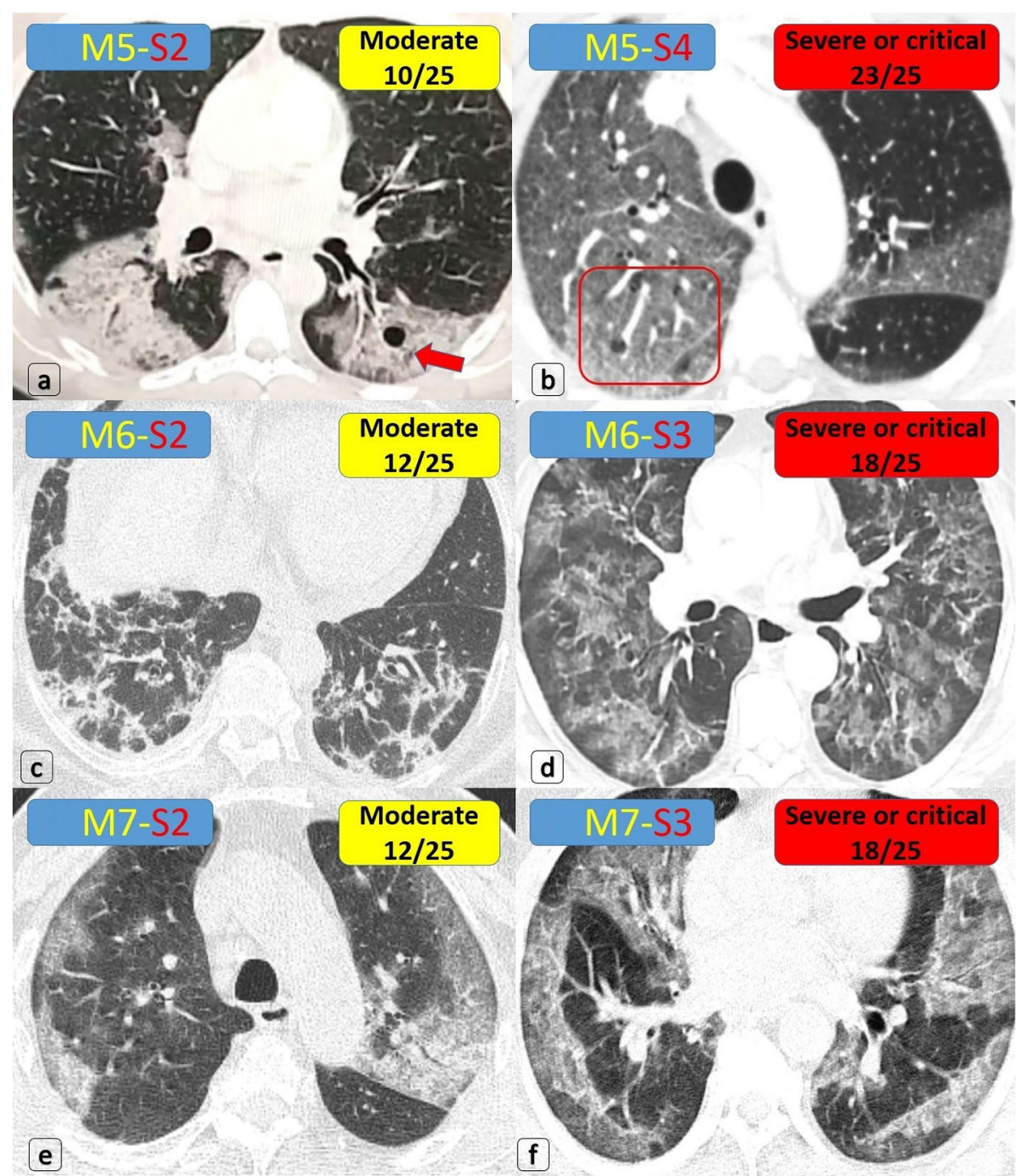

Fig. 6 Multiple patients proved with COVID-19, having variable bilateral moderate/border-line or severe/critical lung involvement. a Bilateral large GGOs (extending $>3 \mathrm{~cm}$ deep from the pleural lining and implicating around 16\% of lung volume) show internal air densities (red arrow) representing "air bubble sign" ... COV [M5-S2] ... CTSI [10/25] ... Moderate degree. b Bilateral large GGOs (involving > 50\% of lung volume) with few "air bubble densities"... COV [M5-S4] ... CTSI [23/25] ... Severe or critical degree. c Bilateral large early secondary fibrotic changes on top of GGOs with parenchymal distortion (around 19\% of lung volume) ... COV [M6-S2] ... CTSI [12/25] ... Moderate degree. d Bilateral large early secondary fibrotic changes on top of GGOs with parenchymal distortion (around 40\% of lung volume) ... COV [M6-S3] ... CTSI [18/25] ... Severe or critical degree. e Bilateral mid-zonal mainly peripheral patchy GGOs with "crazy paving pattern" (involving around $<18 \%$ of lung volume) ... COV [M7-S2] ... CTSI [12/25] ... Moderate degree. f Bilateral multiple variable-sized patchy proximal and peripheral GGOs with "crazy-paving pattern" (around $40 \%$ of lung volume) ... COV [M7-S3] ... CTSI [18/25] ... Severe or critical degree

studies which focused on the relation between the pathological lung volume and clinical severity.

Leonardi et al. [13] reported that the critical value for quantitative lung assessment was $23 \%$. In this study, it was nearly similar (25\%). Still in this study, the authors found that only $33 \%$ of [S3] patients were severe/critical because of an additive effect of early secondary fibrosis patchy crazy-paving.

In this study, the "crazy-paving" pattern was highly associated with clinical severity, this is matching Qin et al.
[16], Lyu et al. [17], and Hu et al. [18] who correlated it to diffuse alveolar damage (DAD). Moreover, the authors found an additive relation of which to the clinical severity among [S2] and [S3] patients.

This study also agreed with Li et al. [14], Zhao W et al. [15], Qin et al. [16]' and $\mathrm{Hu}$ et al. [18] that early fibrosis and architectural distortion accompanied severe patients.

Authors believed that early fibrosis which may occur during the first 10 days of the disease is different from 


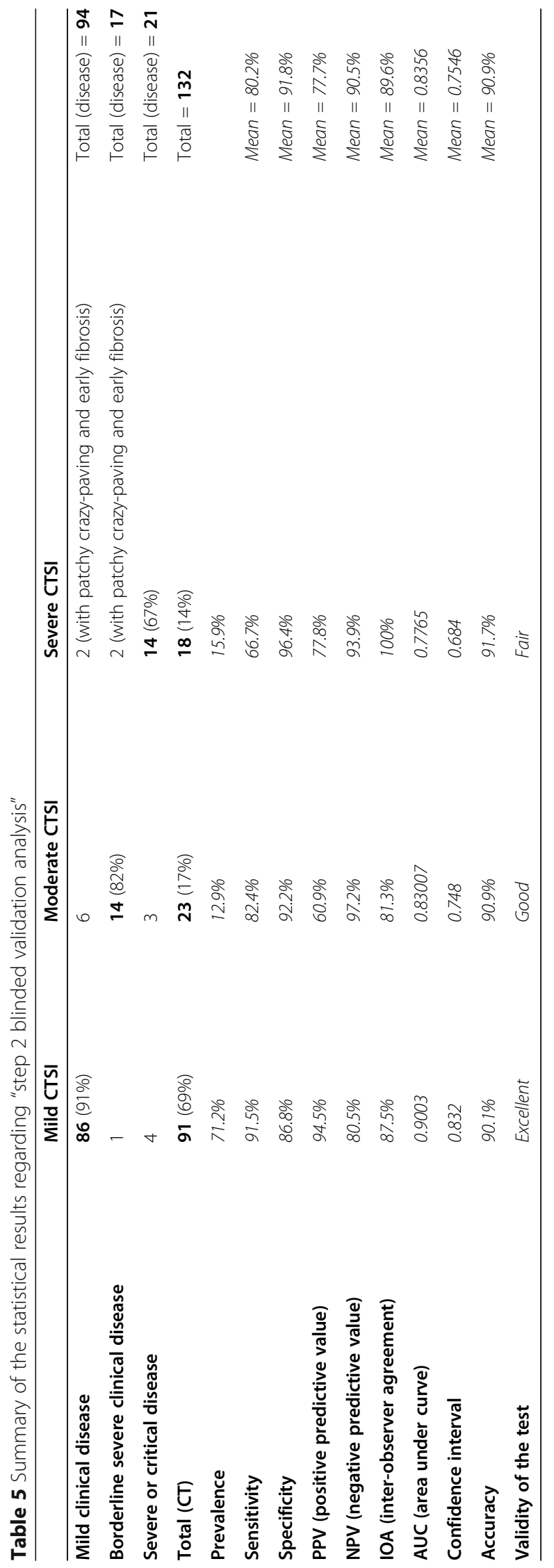




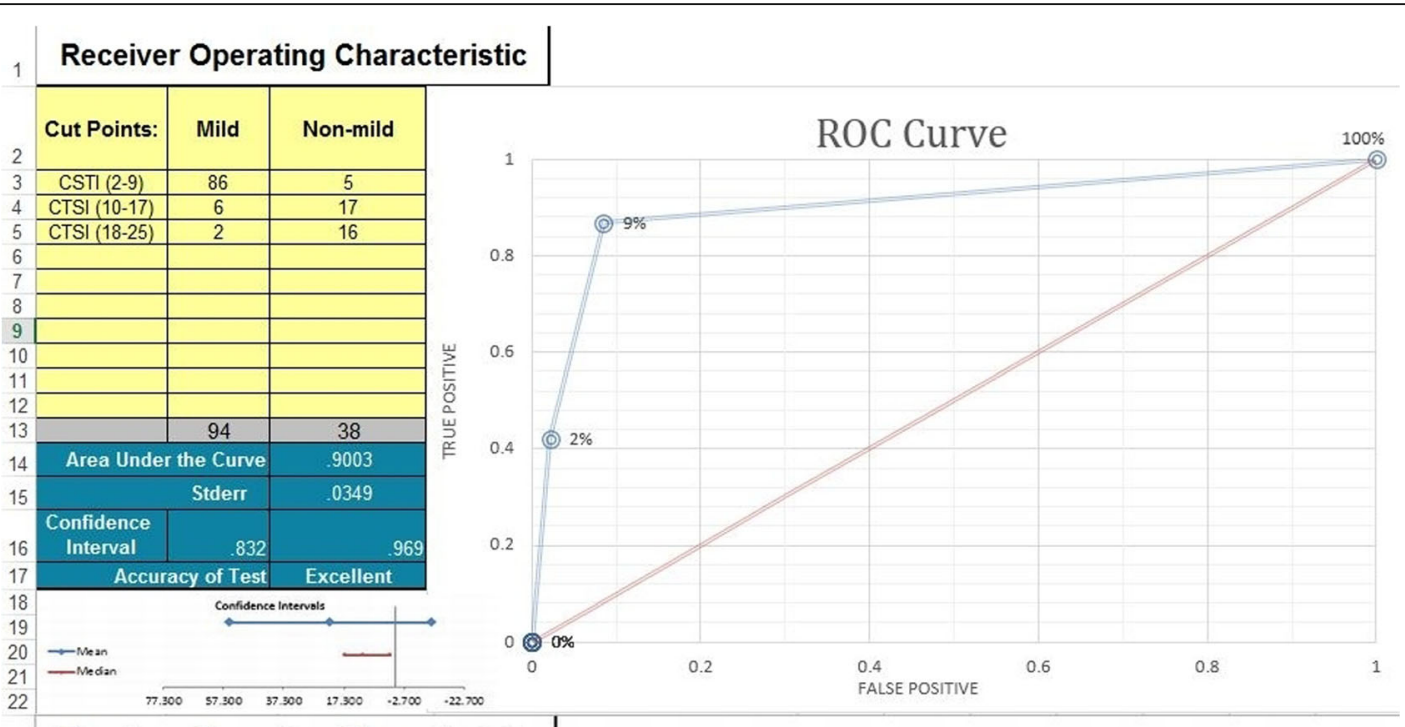

1 Receiver Operating Characteristic

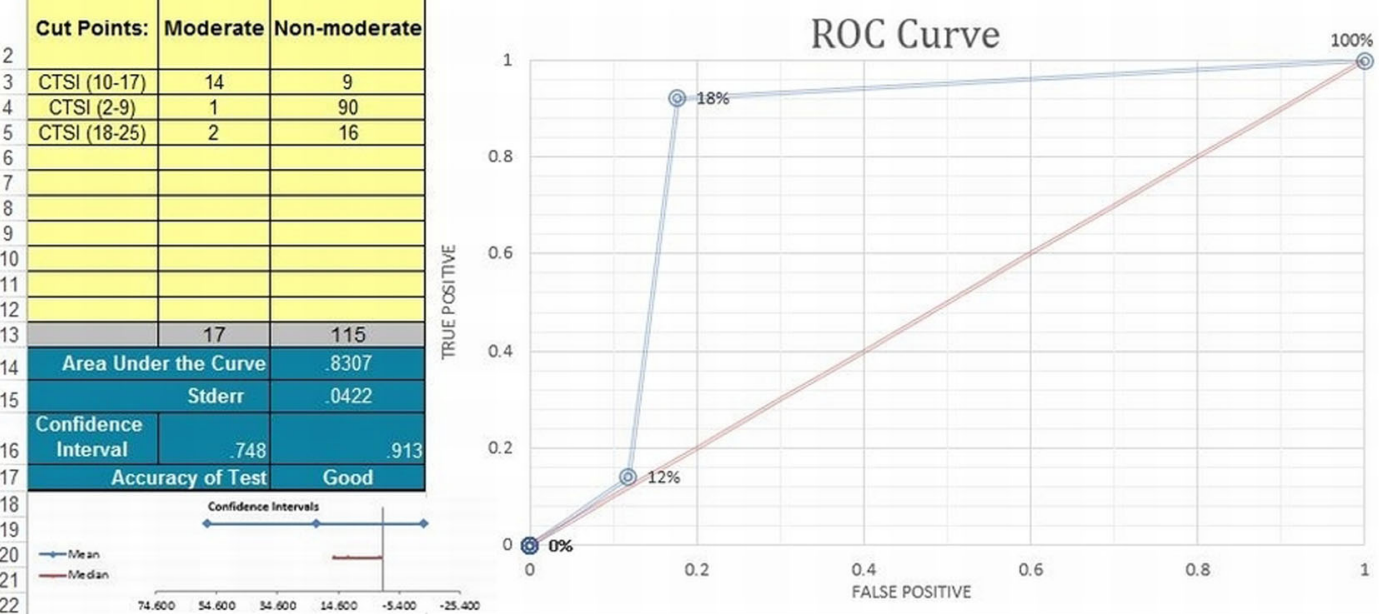

22
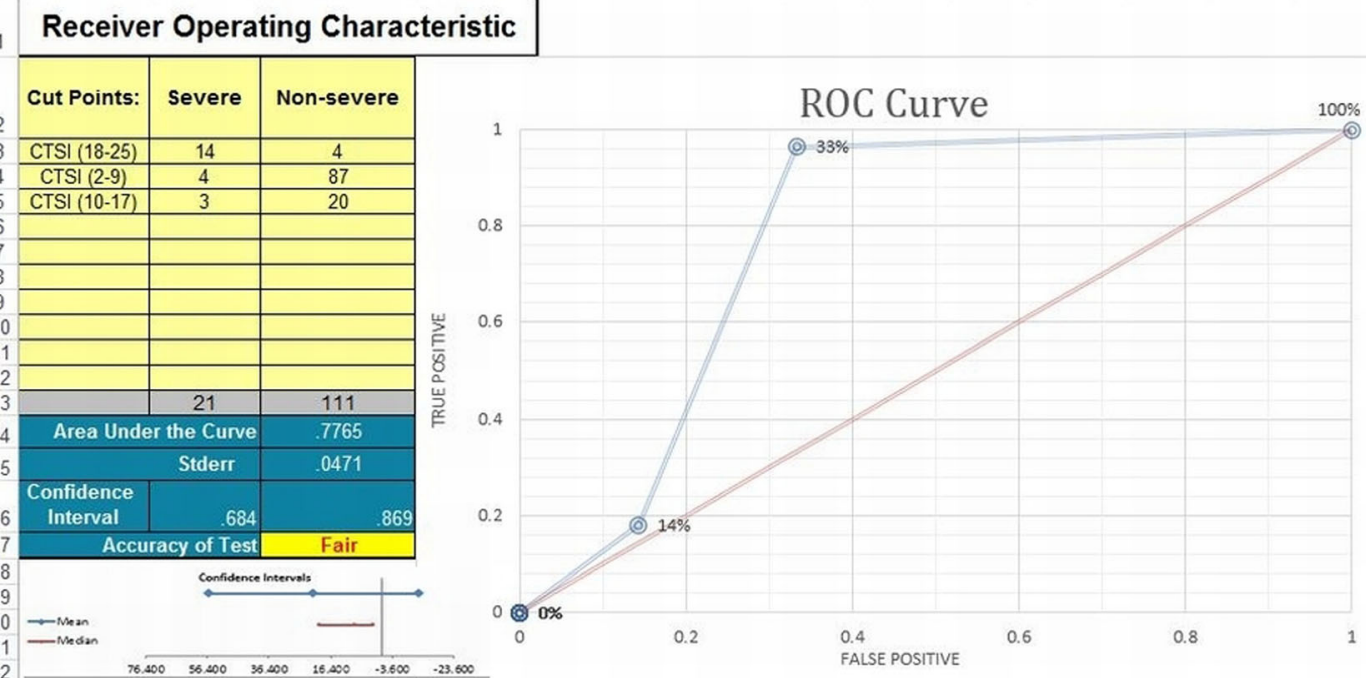

Fig. 7 Receiver operating characteristics (ROC) curves describing the accuracy results for the validation analysis of the proposed CTSI (ranging from fair to excellent) 
delayed fibrosis during the natural healing process. Early fibrosis presents by irregular fibro-atelectatic bands; perpendicular to the pleural surface with diminished volume. While the delayed fibrosis of healing presents by curvilinear bands parallel to the pleural surface with sub-pleural sparing. In this study, early fibro-atelectatic bands with architectural distortion additively increased clinical severity in [S3] patients. This could solve any dilemma between Pan et al. [7] who suggested a good prognosis of fibrosis and Pan et al. [19] as well as Kong et al. [20] who suggested a bad prognosis of which.

Nearly similar to Yee et al. [21], the "air bubble sign" was found among $2 \%$ of our patients. Furthermore, in this study, it was associated with increased severity among [S2] patients. This is mostly explained by associated fibrotic changes.

Also, similar to Zhao et al. [15] and $\mathrm{Hu}$ et al. [18], the severity rate increases in proportionate to the degree of consolidative changes.

The current proposed COVID-19 CTSI expressed accepted results after blinded validation analysis.

This study has the merit that it was conducted on a large group of patients. Meanwhile, the abovementioned studies were all conducted on smaller groups (ranging from 78:150 patients). The overall time for every HRCT evaluation along with CTSI estimation and $\mathrm{CT}$ reporting was also very reasonable and did not exceed $12 \mathrm{~min}$.

However, this study was limited by the low percentage of severe cases in addition to the limited clinical indication/value for CT assessment of mechanically ventilated patients.

\section{Conclusion}

Based on severity analysis, HRCT morphological features should share in CT severity scoring of COVID-19 patients (notably the pulmonary fibrosis and "crazy-paving pattern"). A new optimized CTSI with accepted validation is proposed for the initial staging of COVID-19 patients, using combined morphologic/volumetric assessment instead of the quantitative assessment alone. It could solve the clinico-radiological mismatch among patients with similar quantitative CT results and variable clinical presentation during the absence of pre-existing comorbidities or vascular complications.

\section{Abbreviations \\ COVID 19: Corona virus disease 2019; CTSI: Computed tomography severity index; GGOs: Ground glass opacities; HRCT: High-resolution computed tomography; RT-PCR: Reverse transcription polymerase chain reaction; $\mathrm{O}_{2}$ Sat/Ra: Oxygen saturation in room air; DAD: Diffuse alveolar damage}

\section{Acknowledgements}

The authors would like to acknowledge Prof. Dr. Khaled Matrawy, Prof. Dr. Heba El-Weshahi, Dr. Mohamed Kayed, MD, and Dr. Engy El-Kady, MD for their effort and substantial contribution.

\section{Authors' contributions}

AS (the corresponding author) is responsible for ensuring that the descriptions are accurate and agreed by all authors. AE, TE, and DA had made substantial contributions to all of the following: (1) the conception and design of the radiological work, (2) the acquisition, analysis, and interpretation of radiological data, (3) drafting the work and revising it. HS and Al had made substantial contributions to (1) acquisition, analysis, and interpretation of clinico-laboratory data, and (2) drafting the work and revising it. All authors approved the submitted version. All authors have agreed both to be personally accountable for the authors' own contributions and to ensure that questions related to the accuracy or integrity of any part of the work, even ones in which the authors were not personally involved, are appropriately investigated, resolved, and the resolution documented in the literature. All authors read and approved the final manuscript.

\section{Funding}

None (authors personal contribution).

\section{Availability of data and materials}

The datasets used and/or analyzed during the current study are available from the corresponding author on reasonable request.

\section{Declarations}

\section{Ethics approval and consent to participate}

The medical ethics were considered and respected. The study was approved by Institutional Ethics Committee in Faculty of Medicine, Alexandria University [IRB No.: (00012098), FWA No.: (00018699), Serial No. (0304667)]. Patient consent was waived in this retrospective observational study by the Research Ethics Board, assuring respect of both patient and medical records confidentiality.

\section{Consent for publication \\ Not applicable.}

\section{Competing interests}

The authors declare that they do not have competing interests.

\section{Author details}

${ }^{1}$ Department of Radio-diagnosis, Faculty of Medicine, Alexandria University, Alexandria, Egypt. ${ }^{2}$ Department of Chest Diseases, Faculty of Medicine, Alexandria University, Alexandria, Egypt. ${ }^{3}$ Department of Radiology, Urology and Nephrology Center, Mansoura University, Mansoura, Egypt.

Received: 24 February 2021 Accepted: 30 March 2021

Published online: 15 April 2021

\section{References}

1. Jebril N. World Health Organization declared a pandemic public health menace: A systematic review of the Coronavirus disease 2019 "covid-19", up to 26th March 2020. Available at SSRN 3566298. 2020. https://doi.org/10.213 9/ssrn.3566298

2. Kim H, Hong H, Yoon SH (2020) Diagnostic performance of CT and reverse transcriptase-polymerase chain reaction for Coronavirus disease 2019. Radiology 296(3):E145-E155. https://doi.org/10.1148/radiol.20202 01343

3. Huang C, Wang Y, Li X, Ren L, Zhao J, Hu Y, Zhang L, Fan G, Xu J, Gu X, Cheng Z, Yu T, Xia J, Wei Y, Wu W, Xie X, Yin W, Li H, Liu M, Xiao Y, Gao H, Guo L, Xie J, Wang G, Jiang R, Gao Z, Jin Q, Wang J, Cao B (2020) Clinical features of patients infected with 2019 novel Coronavirus in Wuhan, China. Lancet 395(10223):497-506. https://doi.org/10.1016/ S0140-6736(20)30183-5

4. Dawoud MM, Dawoud TM, Ali NY, Nagy HA (2020) Chest CT in COVID-19 pneumonia: a correlation of lung abnormalities with duration and severity of symptoms. Egypt J Radiol Nuclear Med. 51(1):1-2. https://doi.org/10.11 86/s43055-020-00359-z

5. Sabri YY, Fawzi MM, Nossair EZ, El-Mandooh SM, Hegazy AA, Tadros SF (2020) CT findings of 795 COVID-19 positive cases: a multicenter study in Egypt. Egypt J Radiol Nuclear Med. 51(1):1-9. https://doi.org/10.1186/s43 055-020-00351-7 
6. Ai T, Yang Z, Hou H, Zhan C, Chen C, Lv W, Tao Q, Sun Z, Xia L (2020) Correlation of chest CT and RT-PCR testing in Coronavirus disease 2019 (COVID-19) in China: a report of 1014 cases. Radiology 296(2):E32-E40. https://doi.org/10.1148/radiol.2020200642

7. Pan Y, Guan H, Zhou S, Wang Y, Li Q, Zhu T, Hu Q, Xia L (2020) Initial CT findings and temporal changes in patients with the novel Coronavirus pneumonia (2019-ncov): A study of 63 patients in Wuhan, China. Eur Radiol 30(6):3306-3309. https://doi.org/10.1007/s00330-020-06731-x

8. Bompard F, Monnier H, Saab I, Tordjman M, Abdoul H, Fournier L, Sanchez O, Lorut C, Chassagnon G, Revel MP (2020) Pulmonary embolism in patients with Covid-19 pneumonia. Eur Respir J 56(1):2001365. https://doi.org/10.11 83/13993003.01365-2020

9. Chen LYC, Hoiland RL, Stukas S et al (2020) Confronting the controversy: interleukin-6 and the COVID-19 cytokine storm syndrome. Eur Respir J 56: 2003006. https://doi.org/10.1183/13993003.03006-2020

10. Yang R, Li X, Liu H, Zhen Y, Zhang X, Xiong Q, Luo Y, Gao C, Zeng W (2020) Chest CT severity score: an imaging tool for assessing severe COVID-19. Radiol Cardiothoracic Imaging 2(2):e200047. https://doi.org/1 $0.1148 /$ ryct.2020200047

11. Lessmann N, Sánchez Cl, Beenen L, Boulogne LH, Brink M, Calli E, Charbonnier JP, Dofferhoff T, van Everdingen WM, Gerke PK, Geurts B, Gietema HA, Groeneveld M, van Harten L, Hendrix N, Hendrix W, Huisman HJ, lšgum I, Jacobs C, Kluge R, Kok M, Krdzalic J, LassenSchmidt B, van Leeuwen K, Meakin J, Overkamp M, van Rees Vellinga T, van Rikxoort EM, Samperna R, Schaefer-Prokop C, Schalekamp S, Scholten ET, Sital C, Stöger JL, Teuwen J, Venkadesh KV, de Vente C, Vermaat M, Xie W, de Wilde B, Prokop M, van Ginneken B (2021) Automated assessment of CO-RADS and chest CT Severity scores in patients with suspected COVID-19 using artificial intelligence. Radiology 298(1):239-E28. https://doi.org/10.1148/radiol.2020202439

12. Li K, Fang Y, Li W, Pan C, Qin P, Zhong Y, Liu X, Huang M, Liao Y, Li S (2020) $C T$ image visual quantitative evaluation and clinical classification of coronavirus disease (COVID-19). Eur J Radiol 30(8):4407-4416. https://doi. org/10.1007/s00330-020-06817-6

13. Leonardi A, Scipione R, Alfieri G, Petrillo R, Dolciami M, Ciccarelli F, Perotti S, Cartocci G, Scala A, Imperiale C, lafrate F, Francone M, Catalano C, Ricci P (2020) Role of computed tomography in predicting critical disease in patients with covid-19 pneumonia: a retrospective study using a semiautomatic quantitative method. Eur J Radiol 130:109202. https://doi. org/10.1016/j.ejrad.2020.109202

14. Li K, Wu J, Wu F, Guo D, Chen L, Fang Z, Li C (2020) The clinical and chest $\mathrm{CT}$ features associated with severe and critical COVID-19 pneumonia. Invest Radiol 55(6):00-331. https://doi.org/10.1097/RLI.0000000000000672

15. Zhao W, Zhong Z, Xie X et al (2020) Relation between chest CT findings and clinical conditions of coronavirus disease (COVID-19) pneumonia: a multicenter study. AJR 214:1072-1077. https://doi.org/1 0.2214/AJR.20.22976

16. Qin L, Yang Y, Cao Q, Cheng Z, Wang X, Sun Q, Yan F, Qu J, Yang W (2020) A predictive model and scoring system combining clinical and CT characteristics for the diagnosis of COVID-19. Eur Radiol 30(12):6797-6807. https://doi.org/10.1007/s00330-020-07022-1

17. Lyu P, Liu X, Zhang R, Shi L, Gao J (2020) The performance of chest CT in evaluating the clinical severity of COVID-19 pneumonia: identifying critical cases based on CT characteristics. Invest Radiol 55(7):412-421. https://doi. org/10.1097/RLI.0000000000000689

18. Hu $X$, Zeng $W$, Zhang $Y$, et al. $C T$ imaging features of different clinical types of COVID-19: a Chinese multicenter study. (3/4/2020). https://doi.org/10.213 9/ssrn.3550043.

19. Pan F, Ye T, Sun P (2020) Time course of lung changes on chest $C T$ during recovery from 2019 novel coronavirus (COVID-19) pneumonia. Radiology 295(3):715-721. https://doi.org/10.1148/radiol.2020200370

20. Kong W, Agarwal PP (2020) Chest imaging appearance of COVID-19 infection. Radiol Cardiothoracic Imaging 2(1):e200028. https://doi.org/10.114 8/ryct.2020200028

21. Ye Z, Zhang Y, Wang Y, Huang Z, Song B (2020) Chest CT manifestations of new coronavirus disease 2019 (COVID-19): a pictorial review. Eur Radiol 30(8):4381-4389. https://doi.org/10.1007/s00330-020-06801-0

\section{Publisher's Note}

Springer Nature remains neutral with regard to jurisdictional claims in published maps and institutional affiliations.

\section{Submit your manuscript to a SpringerOpen ${ }^{\circ}$ journal and benefit from:}

- Convenient online submission

- Rigorous peer review

- Open access: articles freely available online

High visibility within the field

- Retaining the copyright to your article

Submit your next manuscript at $\boldsymbol{\nabla}$ springeropen.com 